\title{
Output Feedback NCS of DoS Attacks Triggered by Double-Ended Events
}

\author{
Xinzhi Feng $\mathbb{D},{ }^{1}$ Yang Yang $\mathbb{D},{ }^{1}$ Xiaozhong Qi, ${ }^{1}$ Chunming Xu, ${ }^{2}$ and $Z e ~ \mathbf{J i}^{3}$ \\ ${ }^{1}$ School of Electronic Information Engineering, Changchun University of Science and Technology, Changchun 130022, China \\ ${ }^{2}$ Changchun Shikai Science \& Technology Industry Co. Ltd., Changchun 130012, China \\ ${ }^{3}$ School of Engineering, Cardiff University, Cardiff, UK \\ Correspondence should be addressed to Yang Yang; cloneyang@126.com
}

Received 27 December 2020; Revised 24 January 2021; Accepted 27 January 2021; Published 11 February 2021

Academic Editor: Liguo Zhang

Copyright ( $\odot 2021$ Xinzhi Feng et al. This is an open access article distributed under the Creative Commons Attribution License, which permits unrestricted use, distribution, and reproduction in any medium, provided the original work is properly cited.

In recent years, the research of the network control system under the event triggering mechanism subjected to network attacks has attracted foreign and domestic scholars' wide attention. Among all kinds of network attacks, denial-of-service (DoS) attack is considered the most likely to impact the performance of NCS significantly. The existing results on event triggering do not assess the occurrence of DoS attacks and controller changes, which will reduce the control performance of the addressed system. Aiming at the network control system attacked by DoS, this paper combines double-ended elastic event trigger control, DoS attack, and quantitative feedback control to study the stability of NCS with quantitative feedback of DoS attack triggered by a double-ended elastic event. Simulation examples show that this method can meet the requirements of control performance and counteract the known periodic DoS attacks, which save limited resources and improve the system's antijamming ability.

\section{Introduction}

Scholars have recently devoted much energy to studying network security and control stability of network systems against malicious attacks, including denial of service (DoS) attacks, replay attacks, and spoofing attacks. The research results on different types of network attacks can be obtained in the literature [1-5].

Among all kinds of network attacks, the DoS attack is considered the most likely type of network attack to impact the performance of NCS [6] significantly. In the past few years, the stability analysis of NCS under DoS attacks has been reported in the literature $[7,8]$. Besides, because the event-triggered mechanism can be used to reduce the transmission of information without reducing the overall system performance, in recent years, more and more literature have used the event-triggered method to study the control stability of NCS under DoS attacks [9]. For example, in [6], the author, for the first time, investigated the stability analysis of event-triggered networked linear continuoustime systems under known pulse width modulation DoS attacks. Inspired by this work, the author proposes a more general DoS attack model in [10], in which the DoS attack signal is only constrained by DoS frequency and duration. Some extensions are also reported based on this idea, such as the dynamic output feedback controller [11] and distributed NCS [12].

An event trigger mechanism dependent on the Lyapunov function is proposed for nonlinear continuous systems in [13]. Eqtami et al. [14] use ISS's technology to propose a selftriggering mechanism and a new event triggering mechanism for nonlinear discrete systems. A new event mechanism is proposed for nonlinear systems through Lyapunov in [15]. The asymptotic stability of the system is verified by using the distributed event trigger mechanism in [16], and the small gain theorem for the ISS-Lyapunov function [11] solved the resource consumption and resilient control strategy design of NCS subjected to malicious DoS attacks. In the presence of DoS attacks, the proposed system framework can still guarantee a strictly positive and lower bound on the event time. A system design framework is proposed for the output-based dynamic event trigger control 
(ETC) system under DoS attacks. De Persis and Tesi [17] studied the effective control strategy of nonlinear systems under a DoS attacks and provided the character of the maximum percentage of time that feedback information can be lost without causing feedback instability. It is based on the design of the event's elastic control strategy, and the fixed pattern is used as an exact representation of the interval activity interval. The controller triggered by the event has made a clear representation of the minimum cross-sampling time of the controller. Su et al. [18] proposed incremental algorithms for rapidly evolving the network strategies, and the redundancy rules are further processed.

Focusing on defensive strategies against network attacks in cyberphysical system (CPS), Tian et al. [19] proposed both low- and high-interaction honeypots into CPS as a security management tool deliberately designed to be probed, attacked, and compromised. To improve the attack detection capability of content centric network (CCN), Xu et al. [20] proposed a way of interest flooding attack (IFA) making use of the characteristics of self-similarity of traffic and the information entropy of content name of interest packet.

Distributed Denial-of-Service (DDos) attack has become one of the most destructive network attack. Cheng et al. [21] proposed a detection method of DDos' attacks based on generalized multiple kernel learning combining with the constructed parameter $\mathrm{R}$, and the proposed method can effectively detect DDos' attacks in complex environments with a higher rate and lower error rate. Chen et al. [22] provided the active approach based on the integrated entropy calculations to detect DDos' attack. It is a lightweight approach could be applied in mobile device.

\section{Preparation}

2.1. LMI. The research on LMI (linear matrix inequalities) has been widespread. When it is used to solve control problems, LMI will be used to simplify the practical issues involved. Here are some definitions of LMI and many practical issues of LMI implementation used in later sections [23]. In general, the specific LMI, formula is

$$
G(x)=G_{0}+x_{1} G_{1}+\cdots+x_{m} G_{m}<0,
$$

where $x_{1}, x_{2}, \ldots, x_{m-1}, x_{m}$. is the measure decision variable of LMI. $x=\left(x_{1}, x_{2} \cdots x_{m-1}, x_{m}\right)^{T} \in R^{m}$ is the decision vector $G_{i}=G_{i}^{T} \in R^{n \times n}, \quad i=0,1, \ldots, m$ is a symmetric matrix. Equation (1) is the general form of LMI, but through the exploration of scholars, most of the content of its solution is a variable matrix. If the following Lyapunov inequality

$$
G(X)=X A+A^{T} X+Q<0
$$

where $Q \in R^{n \times n}$ is a real symmetric matrix, $A \in R^{n \times n}$ is a constantly fixed matrix, $X \in R^{n \times n}$ is an unknown solution matrix, so most of the contents of the LMI solution are variable matrices. Formulas (1) and (2) are two representations of LMI, but they embody the same essence. Therefore, the resolution of a kind of LMI problem [24] is obtained as follows: for the existing LMI $G(x)<0$, we can use the function that comes with LMI to solve it, in which there is a function with special meaning, which can be used to test whether the existing $x$ satisfies $G(x)<0$. This kind of case is the LMI technique to solve the problem, and the solver in the function is expressed by feasp.

2.2. $H_{\infty}$ Control Theory. As the core part of robust control, $H_{\infty}$, the theory provides many analysis ideas for control systems and effective solutions for robustness exploration. It can realize the calculation of $H_{\infty}$ the norm. By optimizing this kind of function and then designing the controller reasonably within the prescribed constraint conditions, the robustness of the system becomes very good. The block diagram of $H_{\infty}$ control is shown in Figure 1.

In addition, $u$ is the output information of the controller, $P$ is the controlled object, $w$ is the external interference information, $z$ is the information output of the object, $y$ is the measured value, and $K$ is the controller.

System objects are considered as follows:

$$
\left\{\begin{array}{l}
\dot{x}(t)=\bar{A}_{11} x(t)+\bar{B}_{11} u(t)+\bar{B}_{1 w} w(t), \\
z(t)=\bar{C}_{22} x(t)+\bar{D}_{11} w(t)+\bar{D}_{12} u(t), \\
y(t)=\bar{C}_{33} x(t)+\bar{D}_{31} w(t)+\bar{D}_{32} u(t),
\end{array}\right.
$$

where $u(t) \in R^{m}$ is the input vector of the system, $y(t) \in R^{s}$ is the measured output of the system, $x(t) \in R^{n}$ denotes the state vector, $z(t) \in R^{q}$ represents the control output of the system, and $w(t) \in \mathscr{L}_{2}[0, \infty)$ represents the information of the disturbance signal. $\bar{A}_{11}, \bar{D}_{32}, \bar{B}_{11}, \bar{B}_{1 w}, \bar{C}_{33}, \bar{D}_{21}, \bar{C}_{22}, \bar{D}_{11}$, $\bar{D}_{12}$ is a fixed constant matrix.

For any $\gamma>0$, if formula (3) contains the following properties:

(1) If $w(t)=0$, then the system is stable.

(2) If the function $\Phi(s)$ is used to represent the relationship between $z(t)$ and $w(t)$ by calculating the norm, we have

$$
\|\Phi(s)\|_{\infty}=\sup _{\|w\|_{2} \leq 1} \frac{\|z\|_{2}}{\|w\|_{2}} \leq \gamma^{2} .
$$

And, then it is equal to

$$
\begin{array}{r}
\int_{0}^{\infty} \gamma^{2} w^{T}(t) w(t) \mathrm{d} t \geq \int_{0}^{\infty} z^{T}(t) z(t) \mathrm{d} t \\
\forall w(t) \in \mathscr{L}_{2}[0, \infty) .
\end{array}
$$

Then, system (5) has $H_{\infty}$ performance $\gamma$ [25].

Formula (5) is the ability of anti-interference to the outside world, so $\gamma$ is called the suppression system to the outside world. The smaller the value of $\gamma$ is, the better the control performance of the system will be.

\subsection{Some Lemmas}

Lemma 1. (Jensen inequality). For constant matrices $W \in R^{n \times n}, \quad W=W^{T} \geq 0$, scalar $0 \leq \tau_{1} \leq \tau_{2}$, and vectorvalued functions $\dot{x}:\left[-\tau_{2},-\tau_{1}\right] \longrightarrow R^{n}$. The following formula holds: 


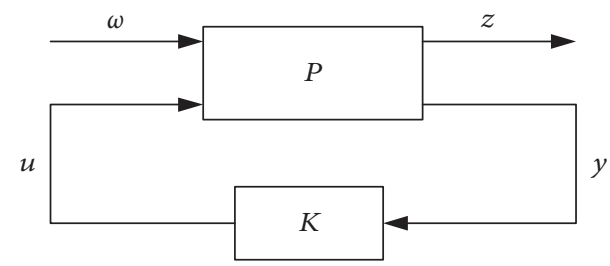

Figure 1: The structure of the $H_{\infty}$ problem.

$$
\begin{aligned}
-\left(\tau_{2}-\tau_{1}\right) \int_{t-\tau_{2}}^{t-\tau_{1}} \dot{x}^{T}(s) W \dot{x}(s) \mathrm{d} s \leq & -\left[\begin{array}{c}
x\left(t-\tau_{1}\right) \\
x\left(t-\tau_{2}\right)
\end{array}\right]^{T} \\
& \cdot\left[\begin{array}{cc}
W & -W \\
-W & W
\end{array}\right] \\
& \cdot\left[\begin{array}{c}
x\left(t-\tau_{1}\right) \\
x\left(t-\tau_{2}\right)
\end{array}\right] .
\end{aligned}
$$

Lemma 2. (Schur complement lemma). For symmetric matrices $W=\left[\begin{array}{cc}W_{1} & * \\ W_{12}^{T} & W_{2}\end{array}\right]$, where $W_{11}$ is $n \times n$ dimensional, "*" is a symmetric term, and the following conditions are equivalent: $W<0, W_{1}<0, W_{2}-W_{12}^{T} W_{1}^{-1} W_{12}<0, W_{2}<0$, and $W_{1}-W_{12} W_{2}^{-1} W_{12}^{T}<0$.

Lemma 3. For the real matrix $\Theta>0, X$ and the given scalar $\delta$, the following formula holds: $\delta^{2} \Theta-2 \delta X \geq-X \Theta^{-1} X$.

\section{Problem Description}

In the current research results, most of the studies are completed under the assumption that the object can be measured. In the actual system, the information of the object cannot be measured directly, so the NCSs' study of state feedback under the DoS attack of event-triggered mechanism cannot act on the system directly. Therefore, the NCSs' research of output feedback under the DoS attack is critical.

3.1. System Description. The model of the system is as follows:

$$
\left\{\begin{array}{l}
\dot{x}_{a}(t)=\left(A_{a}+\Delta A_{a}(t)\right) x_{a}(t)+B_{a} \widehat{u}(t)+B_{w} w(t) \\
y(t)=C_{a} x_{a}(t) \\
z(t)=C_{a 1} x_{a}(t)+D_{a} \widehat{u}(t)+D_{a 1} w(t) \\
x\left(t_{0}\right)=x_{0}
\end{array}\right.
$$

where $\widehat{u}(t) \in R^{m}$ is the input vector, $x_{a}(t) \in R^{n}$ is the state vector, $w(t) \in L_{2}[0, \infty)$ is the interference signal, $y(t) \in R^{q}$ is the measured output, and $A_{a}, B_{a}, C_{a}, C_{a_{1}}, D_{a}, B_{w}, D_{a 1}$ is the constant matrix with appropriate dimension.

$\Delta A_{a}(t)$ matrix is an uncertain matrix and must satisfy the condition of (8):

$$
\Delta A_{a}(t)=D F(t) E,
$$

where $D$ and $E$ is a fixed constant matrix and $F(t)$ is a timevarying unknown matrix with respect to time $t$ is Lebesque and satisfies $F^{T}(t) F(t) \leq I$.

To improve the anti-interference ability of the system in the network, save the limited network resources, and apply the network model attacked by DoS to the control system, the system structure block diagram is shown in Figure 2.

3.2. Establishment of DoS Attack Model. Consider a general attack model that limits the actions of attackers only by limiting the frequency and duration of DoS attacks. It is a kind of periodic network interference signal with energy constraint in the form of pulse width modulation (PWM). Its specific expression is as follows:

$$
P_{\text {Dos }}(t)= \begin{cases}0, & t \in\left[(n-1) T,(n-1) T+T_{\text {off }}\right), \\ 1, & t \in\left[(n-1) T+T_{o f f}, n T\right),\end{cases}
$$

where $n \in N$ is a periodic natural number, $T \in R_{>0}$ is an attack period, $T_{\text {off }} \in R_{>0}\left(T_{\text {off }}<T\right)$ is the zero boundary point of DoS attack and non-DoS attack, and $T_{\text {off }}^{\min }<T_{\text {off }}<T<\infty$. The interval of the network system that is not attacked by DoS is $\left[(n-1) T,(n-1) T+T_{\text {off }}\right)$, and the interval of the network system that is attacked by DoS is $\left[(n-1) T+T_{\text {off }}, n T\right)$.

3.3. Establish a Double-Ended Elastic Event Trigger Mechanism. In view of the situation that the controlled object cannot be measured directly, we propose a two-terminal elastic event trigger mechanism which depends on the information of the object and the controller, which can enhance the anti-interference ability of the system and reduce the amount of data transmitted in the network channel at the same time.

The latest sensor trigger time is expressed by $b_{k} h$, and $b_{k+1} h$ is used to indicate the next trigger time. If there is no DoS attack, the elastic trigger conditions on the sensor side are

$$
\begin{aligned}
b_{k+1} h= & b_{k} h+\min _{q \in N}\left\{q h \mid\left[y\left(b_{k} h\right)-y\left(b_{k} h+q h\right)\right]^{T} W_{y}\right. \\
& \left.\cdot\left[y\left(b_{k} h\right)-y\left(b_{k} h+q h\right)\right] \leq \xi_{y}(t) y^{T}\left(b_{k} h\right) W_{y} y\left(b_{k} h\right)\right\},
\end{aligned}
$$

where $\xi_{y}(t)$ is used to represent the threshold function and $W_{y}>0$ is used to represent the matrix that needs to be solved, and at the same time, it must satisfy

$$
\begin{aligned}
\dot{\xi}_{y}(t)= & \frac{1}{\xi_{y}(t)}\left[\frac{1}{\xi_{y}(t)}-\delta_{y}\right]\left[y\left(b_{k} h\right)-y\left(b_{k} h+q h\right)\right]^{T} W_{y} \\
& \cdot\left[y\left(b_{k} h\right)-y\left(b_{k} h+q h\right)\right],
\end{aligned}
$$

where $\delta_{y} \geq 1$ is the known constant, $y\left(b_{k} h+q h\right)$ the sampling state of the current time, and $y\left(b_{k} h\right)$ shows the latest state of the event trigger.

If there is a DoS attack, the trigger condition of the sensor-side trigger will be defined as 


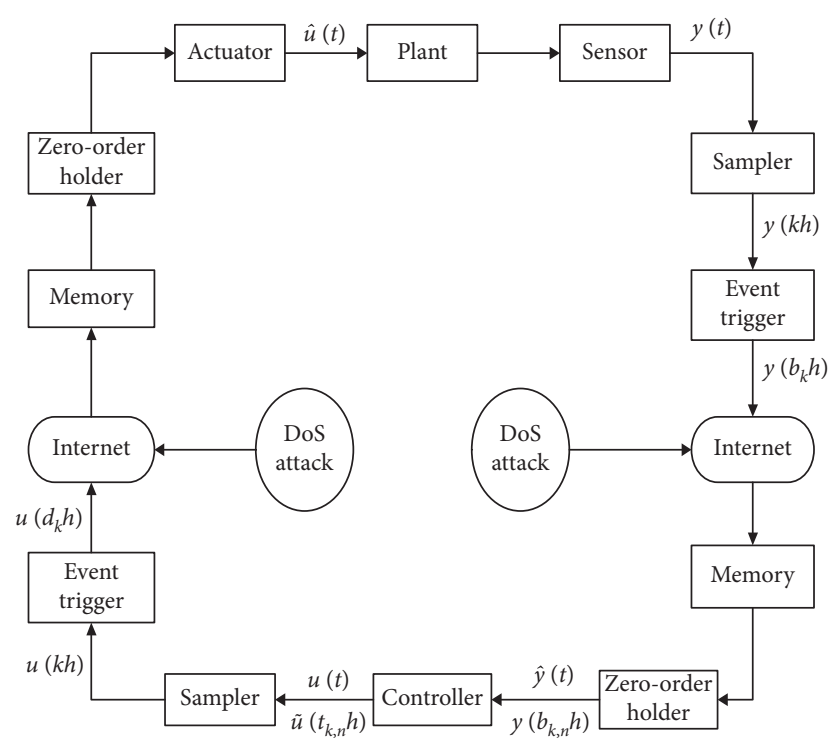

FIGURE 2: DoS attack NCS diagram of double-ended event triggering mechanism under output feedback.

$$
b_{k, n} h=\left\{b_{k_{q}} \mid b_{k_{q}} \in\left[(n-1) T,(n-1) T+T_{o f f}^{\min }\right)\right\} \cup\{n T\}
$$

If the designed elastic event trigger satisfies condition (10) or (12) and the current state $y\left(b_{k} h+q h\right)$ is defined as the latest state $y\left(b_{k+1} h\right)$, then it is sent to the network. $d_{k} h$ indicates the latest time of the controller, and $d_{k+1} h$ indicates the next trigger time. If there is no DoS attack, the elastic trigger conditions of the controller are as follows:

$$
\begin{aligned}
d_{k+1} h= & d_{k} h+\min _{q \in N}\left\{q h \mid\left[u\left(d_{k} h\right)-u\left(d_{k} h+q h\right)\right]^{T} W_{u}\right. \\
& \left.\cdot\left[u\left(d_{k} h\right)-u\left(d_{k} h+q h\right)\right] \leq \xi_{u}(t) u^{T}\left(d_{k} h\right) W_{u} u\left(d_{k} h\right)\right\} .
\end{aligned}
$$

Using $W_{u}>0$ to express the matrix that needs to be solved, it must satisfy

$$
\begin{aligned}
\dot{\xi}_{u}(t)= & \frac{1}{\xi_{u}(t)}\left[\frac{1}{\xi_{u}(t)}-\delta_{u}\right]\left[u\left(d_{k} h\right)-u\left(d_{k} h+q h\right)\right]^{T} W_{u} \\
& \cdot\left[u\left(d_{k} h\right)-u\left(d_{k} h+q h\right)\right] .
\end{aligned}
$$

If there is a DoS attack, the event trigger conditions on the controller side need to be met as follows:

$$
d_{k, n} h=\left\{d_{k_{q}} \mid d_{k_{q}} \in\left[(n-1) T,(n-1) T+T_{o f f}^{\min }\right)\right\} \cup\{n T\} .
$$

3.4. Establish the Model of Closed-Loop System. According to the study of timing and event mechanism in [25], we define $\lambda_{k, n}=b_{k+1}-b_{k}-1$. According to the duration of $\mathrm{ZOH}$, it is finally divided into segments $h$ :

$$
\left[t_{k, n}, t_{k+1, n}\right) h=\bigcup_{l_{k}=0}^{\lambda_{k, n}} Q_{k, n}^{l_{k}}
$$

In addition, $\quad t_{0, n} h=(n-1) T(h<T)$ and $c(n)=\sup$ $\left\{k \in N \mid t_{k}, \quad n h \leq(n-1) T+T_{o f f}^{\min }\right\}$. For $k \in c(n), n \in N$, $\lambda_{k, n}=\inf \left\{m \in N \mid t_{k, n} h+m h \geq t_{k+1, n} h \geq n T\right\}$ :

$\left\{\begin{array}{l}C_{1, n} \hat{=}\left[(n-1) T,(n-1) T+T_{o f f}\right), \vartheta(n) \hat{=}\{0,1, \ldots, k(n)\}, \\ C_{2, n} \hat{=}\left[(n-1) T+T_{o f f}, n T\right), Q_{k, n}^{l_{k}} \hat{=}\left[t_{k, n}, t_{k+1, n}\right) h, k \in \vartheta(n),\end{array}\right.$

$$
\begin{gathered}
Q_{k, n}^{l_{k}}= \begin{cases}{\left[t_{k, n}, t_{k, n}+h\right) h,} & l_{k}=0, \\
{\left[t_{k, n}+h, t_{k, n}+2 h\right) h,} & l_{k}=1, \\
\vdots & \left.t_{k, n}+\left(\lambda_{k}-1\right) h, t_{k, n}+\lambda_{k} h\right) h, \\
{\left[t_{k, n}+\lambda_{k} h, t_{k+1, n}\right) h,} & l_{k}=\lambda_{k}-1,\end{cases} \\
\begin{cases}\Upsilon_{k}^{m}=\left[t_{k, n} h+(m-1) h, t_{k, n} h+m h\right), & l_{k}=\lambda_{k}, \\
\Upsilon_{k}^{\lambda_{k, n}}=\left[t_{k, n} h+\left(\lambda_{k, n}-1\right) h,\right. & \left.t_{k+1, n} h\right),\end{cases}
\end{gathered}
$$

$$
C_{1, n}=\bigcup_{k=0}^{k(n)}\left\{Q_{k, n}^{l_{k}} \cap C_{1, n}\right\} \subseteq \bigcup_{k=0}^{k(n)} Q_{k, n}^{l_{k}} .
$$

The combination of (18)-(20) can be described as follows:

$$
C_{1, n}=\bigcup_{k=0}^{k(n)} \bigcup_{m=1}^{\lambda_{k, n}}\left\{\Upsilon_{k}^{m} \cap C_{1, n}\right\}
$$

Let $\psi_{k}^{m}=\Upsilon_{k}^{m} \cap C_{1, n}$, i.e., $C_{1, n}=\cup_{k=0}^{k(n)} \cup_{m=1}^{\lambda_{k, n}} \psi_{k}^{m}$.

Define the function, $\eta(t)=t-\left(b_{k, n} h+l_{k} h\right), t \in \psi_{k}^{m}$, i.e.,

$$
\eta(t)= \begin{cases}t-b_{k, n} h, & t \in \psi_{k}^{1}, \\ t-\left(b_{k . n} h+h\right), & t \in \psi_{k}^{2}, \\ \vdots & \\ t-\left(b_{k, n} h+\lambda_{k} h\right), & t \in \psi_{k}^{\lambda_{k}-1},\end{cases}
$$

where $\tau \leq \eta(1) \leq \eta(t) \leq \eta(2)=h+\tau, \dot{\eta}(t)=1, \quad t \in \psi_{k}^{m}$.

The set of real-time update times of the feedback forward channel zero-order holder will be expressed as $\left\{\tau, t_{1, n}, t_{2, n}, \ldots\right\}$, where $t_{k, n}=b_{k, n} h+\tau$. According to the relevant properties of $\mathrm{ZOH}$, the input information of output feedback is

$$
\widehat{y}(t)=y\left(b_{k, n} h\right), \quad t \in\left[t_{k, n}, t_{k+1, n}\right) h .
$$

The control input of the controlled plant (7) is

$$
\widehat{u}(t)=u\left(\bar{d}_{k, n} h\right), \quad t \in \psi_{k}^{m} .
$$


Several functions are defined as follows.

(1) Define the function:

$$
e_{y}(t)=y\left(b_{k, n} h\right)-y\left(b_{k, n} h+l_{k} h\right), \quad t \in \psi_{k}^{m} .
$$

According to formulas (22), (23), and (25), the controller inputs as

$$
\hat{y}(t)=y\left(b_{k, n} h\right)=\left[e_{y}(t)+y(t-\eta(t))\right], \quad t \in \psi_{k}^{m} \text {. }
$$

(2) Define the function:

$$
e_{u}(t)=u\left(\bar{d}_{k, n} h\right)-u\left(b_{k, n} h+l_{k} h\right), \quad t \in \psi_{k}^{m} .
$$

According to formulas (22), (24), and (25), the control input of the controlled object (7) is

$$
\widehat{u}(t)=u\left(\bar{d}_{k, n} h\right)=e_{u}(t)+u(t-\eta(t)), \quad t \in \psi_{k}^{m} .
$$

The feedback controller for output dynamics is

$$
\left\{\begin{array}{l}
\dot{x}(t)=A_{c} x_{c}(t)+A_{c d} x_{c}(t-\eta(t))+B_{c} \hat{y}(t), \\
u(t)=K x_{c}(t), \quad t \in \psi_{k}^{m},
\end{array}\right.
$$

where $\hat{y}(t)$ is the input vector of dynamic output feedback, $u(t)$ is the output vector, and $x_{c}(t) \in R^{n}$ matrix is the matrix with a proper dimension of the state vector $A_{c}, A_{c d}, K, B_{c}$.

In the same timing, the input $\widehat{u}(t)$ of (29) is substituted into model (7), and the input $\hat{y}(t)$ of (26) is brought into (29). The final closed-loop model is

$$
\left\{\begin{array}{l}
\left\{\begin{array}{l}
\dot{\varepsilon}(t)=A_{1} \varepsilon(t)+A_{12} \varepsilon(t-\eta(t))+B_{11} e_{y}(t)+B_{12} e_{u}(t)+\bar{B}_{w} w(t), \\
z(t)=\bar{C}_{a 1} \varepsilon(t)+D_{a} e_{u}(t)+D_{a} K \varepsilon(t-\eta(t))+D_{a 1} w(t), \quad t \in Q_{k, n}^{l_{k}} \cap C_{1, n}
\end{array}\right. \\
\left\{\begin{array}{l}
\dot{\varepsilon}(t)=A_{1} \varepsilon(t)+A_{22} \varepsilon(t-\eta(t))+B_{11} e_{y}(t)+\bar{B}_{w} w(t), \\
z(t)=\bar{C}_{a 1} \varepsilon(t)+D_{a 1} w(t), \quad t \in C_{2, n} .
\end{array}\right.
\end{array}\right.
$$

In order to facilitate the analysis, this chapter introduces the switching signal:

$$
\psi(t)= \begin{cases}1, & t \in[-h, 0) \cup\left(\underset{n \in N}{\cup} C_{1, n}\right), \\ 2, & t \in \underset{n \in N}{\cup} C_{2, n} .\end{cases}
$$

For $\psi(t)=i \in\{1,2\}$ and $n \in N$, define

$$
t_{i, n}=\left\{\begin{array}{l}
(n-1) T, \quad i=1, \\
(n-1) T+T_{o f f}^{\min }, \quad i=2 .
\end{array}\right.
$$

Let $C_{i, n}=\left[t_{i, n}, t_{3-i, n+i-1}\right), \quad \psi\left(t_{i, n}\right)=i, \psi\left(t_{i, n}^{-}\right)=3-i$.

Based on the set switching signal $\psi(t)$, the closed-loop system (30) can be simplified as follows:

$$
\left\{\begin{array}{l}
\dot{\varepsilon}_{\psi(t)}(t)=A_{1} \varepsilon(t)+A_{i 2} \varepsilon(t-\eta(t))+B_{11} e_{y}(t)+B_{i 2} e_{u}(t)+\bar{B}_{w} w(t), \\
i \in\{1,2\}, \quad t \in \cup_{n \in N}\left[t_{i, n}, t_{3-i, n+i-1}\right), \\
z(t)=\bar{C}_{a 1} \varepsilon(t)+D_{a 1} w(t)+D_{i a} e_{u}(t)+D_{i a} K \varepsilon(t-\eta(t)), \\
x\left(t_{0}\right)=x_{0},
\end{array}\right.
$$

where

$$
\begin{aligned}
D_{1 a} & =D_{a}, \\
D_{2 a} & =0, \\
B_{22} & =0, \\
\bar{C}_{a 1} & =\left[\begin{array}{ll}
C_{a 1} & 0
\end{array}\right], \\
\mathcal{E}(t) & =\left[\begin{array}{c}
x_{a}(t) \\
x_{c}(t)
\end{array}\right], \\
\bar{B}_{w} & =\left[\begin{array}{c}
B_{w} \\
0
\end{array}\right], \\
B_{11} & =\left[\begin{array}{c}
0 \\
B_{c}
\end{array}\right], \\
B_{12} & =\left[\begin{array}{c}
B_{a} \\
0
\end{array}\right], \\
A_{1} & =\left[\begin{array}{cc}
A_{a}+\Delta A_{a}(t) & 0 \\
0 & A_{c}
\end{array}\right], \\
A_{12} & =\left[\begin{array}{cc}
0 & B_{a} K \\
B_{c} C_{a} & A_{c d}
\end{array}\right], \\
A_{22} & =\left[\begin{array}{cc}
0 & 0 \\
B_{c} C_{a} & A_{c d}
\end{array}\right] .
\end{aligned}
$$




\section{Stability Analysis of Closed-Loop System}

Through the LMI method and the related theory of Lyapunov, aiming at whether the networked control system is attacked by DoS or not in the unmeasurable state, based on the proposed two-terminal elastic event trigger mechanism, the exponential stability of the system is proved.

Theorem 1. For a given state gain matrix $K$ and interference signal $P_{D o S}(t)(9)$, the parameters $T$ and $T_{\text {off }}^{\min }$ are known. Consider system (33), for the known positive scalar $\alpha_{i}, h>0$ and $\gamma>0$ and the double-ended elastic event trigger parameter $0<\delta_{y}<1$ and $0<\delta_{u}<1$, if there is a positive definite matrix $W_{u}, W_{y}, P_{i}, Q_{i}, R_{i}$, and $Z_{i}$ and the matrix $M_{i}, N_{i}, S_{i}, i \in\{1,2\}$, while satisfying the following conditions

$$
\begin{aligned}
\sum_{i} & =\left[\begin{array}{cccccc}
\Pi_{11}^{i} & * & * & * & * & * \\
\sqrt{h} N_{i}^{T} & \Upsilon_{22}^{i} & * & * & * & * \\
\sqrt{h} S_{i}^{T} & 0 & \Upsilon_{33}^{i} & * & * & * \\
\sqrt{h} M_{i}^{T} & 0 & 0 & \Upsilon_{44}^{i} & * & * \\
\sqrt{h} R_{i} \bar{A}_{i} & 0 & 0 & 0 & \Upsilon_{55}^{i} & * \\
\sqrt{h} Z_{i} \bar{A}_{i} & 0 & 0 & 0 & 0 & \Upsilon_{66}^{i}
\end{array}\right]<0, \quad i=1,2, \\
\Pi_{11}^{i} & =\Phi_{i 1}+\Phi_{i}+\Phi_{i}^{T}, \\
\Upsilon_{22}^{i} & =\Upsilon_{33}^{i} \\
\Upsilon_{44}^{i} & =-e^{2(-1)^{i} \alpha_{i} \lambda_{i} h} Z_{i}, \\
\lambda_{1} & =1, \\
\lambda_{2} & =0 \\
\Upsilon_{55}^{i} & =-R_{i}, \\
\Upsilon_{66}^{i} & =-Z_{i},
\end{aligned}
$$

then along the system trajectories (4)-(21), for any $n \in N$, there are

$$
V_{i}(t) \leq e^{2(-1)^{i} \alpha_{i}\left(t-t_{i, n}\right)} V_{i}\left(t_{i, n}\right), \quad t \in\left[t_{i, n}, t_{3-i, n+i-1}\right) .
$$

Proof. Select the following time-varying Lyapunov functions:

$$
\begin{aligned}
V_{\psi(t)}(t)= & \varepsilon^{T}(t) P_{\psi(t)} \varepsilon(t)+\int_{t-h}^{t} \varepsilon^{T}(s) \exp (\bullet) Q_{\psi(t)} \varepsilon(s) \mathrm{d} s \\
& +\int_{-h}^{0} \int_{t+\theta}^{t} \dot{\varepsilon}^{T}(s) \exp (\bullet) Z_{\psi(t)} \dot{\varepsilon}(s) \mathrm{d} s \mathrm{~d} \theta \\
& +\int_{-h}^{0} \int_{t+\theta}^{t} \dot{\varepsilon}^{T}(s) \exp (\bullet) R_{\psi(t)} \dot{\varepsilon}(s) \mathrm{d} s \mathrm{~d} \theta \\
& +\frac{1}{2} \xi_{y}^{2}(t)+\frac{1}{2} \xi_{u}^{2}(t) .
\end{aligned}
$$

When $P_{\psi(t)}>0, R_{\psi(t)}>0, Q_{\psi(t)}>0, Z_{\psi(t)}>0, \alpha_{\psi(t)}>0$, and $\exp (\bullet)=e^{2(-1) \psi(t) \alpha_{\psi(t)}(t-s)}$ are given, for any $k \in \vartheta(n), n \in N$, when $\psi(t)=1$, calculate the derivative $V_{1}(t)$ at $t \in Q_{k, n}^{l_{k}} \cap C_{1, n}$ :

$$
\begin{aligned}
\dot{V}_{1}(t) \leq & -2 \alpha_{1} V_{1}(t)+2 \alpha_{1} \varepsilon^{T}(t) P_{1} \varepsilon(t)+2 \varepsilon^{T}(t) P_{1} \dot{\varepsilon}(t)+\varepsilon^{T}(t) Q_{1} \varepsilon(t) \\
& -\varepsilon^{T}(t-h) e^{-2 \alpha_{1} h} Q_{1} \varepsilon(t-h)+h \dot{\varepsilon}^{T}(t)\left(R_{1}+Z_{1}\right) \dot{\varepsilon}(t) \\
& -\int_{t-h}^{t} \dot{\varepsilon}^{T}(s) e^{-2 \alpha_{1} h} Z_{1} \dot{\varepsilon}(s) \mathrm{d} s-\int_{t-\eta(t)}^{t} \dot{\varepsilon}^{T}(s) e^{-2 \alpha_{1} h} R_{1} \dot{\varepsilon}(s) \mathrm{d} s \\
& +2 \widetilde{\varepsilon}^{T}(t) M_{1}\left[\varepsilon(t)-\varepsilon(t-h)-\int_{t-h}^{t} \dot{\varepsilon}(s) \mathrm{d} s\right] \\
& +2 \widetilde{\varepsilon}^{T}(t) N_{1}\left[\varepsilon(t)-\varepsilon(t-\eta(t))-\int_{t-\eta(t)}^{t} \dot{\varepsilon}(s) \mathrm{d} s\right] \\
& +2 \widetilde{\varepsilon}^{T}(t) S_{1}\left[\varepsilon(t-\eta(t))-\varepsilon(t-h)-\int_{t-h}^{t-\eta(t)} \dot{\varepsilon}(s) \mathrm{d} s\right] \\
& -\int_{t-h}^{t-\eta(t)} \dot{\varepsilon}^{T}(s) e^{-2 \alpha_{1} h} R_{1} \dot{\varepsilon}(s) \mathrm{d} s+\xi_{y}(t) \dot{\xi}_{y}(t)+\xi_{u}(t) \dot{\xi}_{u}(t), \quad t \in Q_{k, n}^{l_{k}} \cap C_{1, n} .
\end{aligned}
$$


According to the literature [25], we can obtain

$$
\begin{aligned}
& -2 \widetilde{\mathcal{\varepsilon}}^{T}(t) M_{1} \int_{t-h}^{t} \dot{\varepsilon}(s) \mathrm{d} s \leq h \widetilde{\mathcal{\varepsilon}}^{T}(t) M_{1} e^{2 \alpha_{1} h} Z_{1}^{-1} M_{1}^{T} \widetilde{\mathcal{\varepsilon}}(t)+\int_{t-h}^{t} \dot{\varepsilon}^{T}(s) e^{-2 \alpha_{1} h} Z_{1} \dot{\varepsilon}(s) \mathrm{d} s, \\
& -2 \widetilde{\mathcal{E}}^{T}(t) N_{1} \int_{t-\eta(t)}^{t} \dot{\varepsilon}(s) \mathrm{d} s \leq h \widetilde{\mathcal{\varepsilon}}^{T}(t) N_{1} e^{2 \alpha_{1} h} R_{1}^{-1} N_{1}^{T} \widetilde{\mathcal{\varepsilon}}(t)+\int_{t-\eta(t)}^{t} \dot{\varepsilon}^{T}(s) e^{-2 \alpha_{1} h} R_{1} \dot{\varepsilon}(s) \mathrm{d} s, \\
& -2 \widetilde{\mathcal{\varepsilon}}^{T}(t) S_{1} \int_{t-h}^{t-\eta(t)} \dot{\varepsilon}(s) \mathrm{d} s \leq h \widetilde{\mathcal{\varepsilon}}^{T}(t) S_{1} e^{2 \alpha_{1} h} R_{1}^{-1} S_{1}^{T} \widetilde{\mathcal{\varepsilon}}(t)+\int_{t-h}^{t-\eta(t)} \dot{\varepsilon}^{T}(s) e^{-2 \alpha_{1} h} R_{1} \dot{\varepsilon}(s) \mathrm{d} s .
\end{aligned}
$$

The simplified expressions of the two-terminal elastic event-triggering mechanisms (10)-(13) and trigger functions (23) and (28) can be obtained from the same time sequence $\psi_{k}^{m}$ of the input $\hat{y}(t)$ of equation (23) and the object input $\widehat{u}(t)$ of $(28)$ :

$$
\begin{aligned}
\frac{1}{\xi_{y}(t)} e_{y}^{T}(t) W_{y} e_{y}(t) \leq & {\left[e_{y}(t)+y(t-\eta(t))\right]^{T} W_{y} } \\
& \cdot\left[e_{y}(t)+y(t-\eta(t))\right], \quad t \in Q_{k, n}^{l_{k}} \cap C_{1, n},
\end{aligned}
$$

$$
\begin{aligned}
\xi_{y}(t) \dot{\xi}_{y}(t) & =\left[\frac{1}{\xi_{y}(t)}-\delta_{y}\right] e_{y}^{T}(t) W_{y} e_{y}(t) \\
& =\frac{1}{\xi_{y}(t)} e_{y}^{T}(t) W_{y} e_{y}(t)-\delta_{y} e_{y}^{T}(t) W_{y} e_{y}(t) \\
& \leq\left[e_{y}(t)+y(t-\eta(t))\right]^{T} W_{y}\left[e_{y}(t)+y(t-\eta(t))\right] \\
& -\delta_{y} e_{y}^{T}(t) W_{y} e_{y}(t), \quad t \in Q_{k, n}^{l_{k}} \cap C_{1, n},
\end{aligned}
$$

$$
\begin{aligned}
\frac{1}{\xi_{u}(t)} e_{u}^{T}(t) W_{u} e_{u}(t) \leq & {\left[e_{u}(t)+u(t-\eta(t))\right]^{T} W_{u} } \\
& \cdot\left[e_{u}(t)+u(t-\eta(t))\right], \quad t \in Q_{k, n}^{l_{k}} \cap C_{1, n},
\end{aligned}
$$$$
\begin{aligned}
\xi_{u}(t) \dot{\xi}_{u}(t)= & {\left[\frac{1}{\xi_{u}(t)}-\delta_{u}\right] e_{u}^{T}(t) W_{u} e_{u}(t) } \\
= & \frac{1}{\xi_{u}(t)} e_{u}^{T}(t) W_{u} e_{u}(t)-\delta_{u} e_{u}^{T}(t) W_{u} e_{u}(t) \\
\leq & {\left[e_{u}(t)+u(t-\eta(t))\right]^{T} W_{u}\left[e_{u}(t)+u(t-\eta(t))\right] } \\
& -\delta_{u} e_{u}^{T}(t) W_{u} e_{u}(t), \quad t \in Q_{k, n}^{l_{k}} \cap C_{1, n} .
\end{aligned}
$$

Combining formulas (38)-(41), (43), and (45),

$$
\begin{aligned}
\dot{V}_{1}(t) \leq & -2 \alpha_{1} V_{1}(t)+\chi^{T}(t)\left[\Pi_{111}^{1}+h N_{1} e^{2 \alpha_{1} h} R_{1}^{-1} N_{1}^{T}\right. \\
& +h M_{1} e^{2 \alpha_{1} h} Z_{1}^{-1} M_{1}^{T}+h S_{1} e^{2 \alpha_{1} h} R_{1}^{-1} S_{1}^{T} \\
& \left.+h \bar{A}_{1}^{T}\left(R_{1}+Z_{1}\right) \bar{A}_{1}\right] \chi(t),
\end{aligned}
$$

where $\chi(t)=\left\{\varepsilon(t), \varepsilon(t-\eta(t)), \varepsilon(t-h), e_{y}(t), e_{u}(t), w(t)\right\}$. Apparently,

$$
\begin{aligned}
\dot{V}_{1}(t)= & \dot{V}_{1}(t)+\gamma^{2} w(t) w^{T}(t)-\gamma^{2} w(t) w^{T}(t) \\
& -z(t) z^{T}(t)+z(t) z^{T}(t) .
\end{aligned}
$$

$z(t)$ of (29) in the closed-loop system is brought into (47) to obtain (48):

$$
\begin{aligned}
& \dot{V}_{1}(t) \leq-2 \alpha_{1} V_{1}(t)+\chi^{T}(t)\left[\Pi_{11}^{1}+h N_{1} e^{2 \alpha_{1} h} R_{1}^{-1} N_{1}^{T}+h M_{1} e^{2 \alpha_{1} h} Z_{1}^{-1} M_{1}^{T}+h S_{1} e^{2 \alpha_{1} h} R_{1}^{-1} S_{1}^{T}+h \bar{A}_{1}^{T}\left(R_{1}+Z_{1}\right) \bar{A}_{1}\right] \chi \\
& \cdot(t)+\gamma^{2} w^{T}(t) w(t)-z^{T}(t) z(t) \\
& \Phi_{11}=\left[\begin{array}{cccccc}
P_{11} & * & * & * & * & * \\
A_{12}^{T} P_{1}+K^{T} D_{a}^{T} C_{a 1} & H_{221} & * & * & * & * \\
0 & 0 & -e^{-2 \alpha_{1} h} Q_{1} & * & * & * \\
B_{11}^{T} P_{1} & W_{y} C_{a} & 0 & W_{y}-\delta_{y} W_{y} & * & * \\
D_{a}^{T} C_{a 1}+B_{12}^{T} P_{1} & H_{52} & 0 & 0 & H_{551} & * \\
\bar{B}_{w}^{T} P_{1}+D_{a 1}^{T} C_{a 1} & D_{a 1}^{T} D_{a} K & 0 & 0 & D_{a 1}^{T} D_{a} & D_{a 1}^{T} D_{a 1}-\gamma^{2}
\end{array}\right] \text {, } \\
& P_{11}=P_{1} A_{1}+A_{1}^{T} P_{1}+Q_{1}+2 \alpha_{1} P_{1}+C_{a 1}^{T} * C_{a 1}, H_{551}=-\delta_{u} W_{u}+W_{u}+D_{a}^{T} D_{a} \text {, } \\
& \Phi_{1}=\left[\begin{array}{llll}
M_{1}+N_{1}-N_{1}+S_{1}-M_{1}-S_{1} & 0 & 0 & 0
\end{array}\right] \text {, } \\
& \bar{A}_{1}=\left[\begin{array}{llllll}
A_{1} & A_{12} & 0 & B_{11} & B_{12} & \bar{B}_{w}
\end{array}\right] \text {, } \\
& H_{221}=K^{T} W_{u} K+C_{a}^{T} W_{y} C_{a}+K^{T} D_{a}^{T} D_{a} K, H_{52}=W_{u} K+D_{a}^{T} D_{a} K \text {. }
\end{aligned}
$$


By using Shur Lemma, the following results are obtained:

$$
\begin{gathered}
\Pi_{11}^{1}+h N_{1} e^{2 \alpha_{1} h} R_{1}^{-1} N_{1}^{T}+h M_{1} e^{2 \alpha_{1} h} Z_{1}^{-1} M_{1}^{T} \\
\quad+h S_{1} e^{2 \alpha_{1} h} R_{1}^{-1} S_{1}^{T}+h \bar{A}_{1}^{T}\left(R_{1}+Z_{1}\right) \bar{A}_{1}<0 .
\end{gathered}
$$

On the contrary, when $t \in\left[t_{2, n}, t_{1, n+1}\right)$, the same result is obtained:

$$
\begin{aligned}
& \dot{V}_{2}(t) \leq 2 \alpha_{2} V_{2}(t)+\chi^{T}(t)\left[\Pi_{11}^{2}+h N_{2} R_{2}^{-1} N_{2}^{T}+h M_{2} Z_{2}^{-1} M_{2}^{T}+h S_{2} R_{2}^{-1} S_{2}^{T}+h S_{2} R_{2}^{-1} S_{2}^{T}+h \bar{A}_{2}^{T}\left(R_{2}+Z_{2}\right) \bar{A}_{2}\right] \chi \\
& \cdot(t)+\gamma^{2} w^{T}(t) w(t)-z^{T}(t) z(t), \\
& \Phi_{21}=\left[\begin{array}{cccccc}
P_{2} A_{1}+A_{1}^{T} P_{2}+2 \alpha_{2} P_{2}+Q_{2}+C_{a 1}^{T} * C_{a 1} & * & * & * & * & * \\
A_{22}^{T} P_{2} & H_{222} & * & * & * & * \\
0 & 0 & -Q_{2} & * & * & * \\
B_{11}^{T} P_{2} & W_{y} C_{a} & 0 & W_{y}-\delta_{y} W_{y} & * & * \\
0 & W_{u} K & 0 & 0 & H_{552} & * \\
\bar{B}_{w}^{T} P_{2}+D_{a 1}^{T} C_{a 1} & 0 & 0 & 0 & 0 & D_{a 1}^{T} D_{a 1}-\gamma^{2}
\end{array}\right] \text {, } \\
& H_{222}=K^{T} W_{u} K+C_{a}^{T} W_{y} C_{a} \text {, } \\
& H_{552}=-\delta_{u} W_{u}+W_{u} \text {, } \\
& \bar{A}_{2}=\left[\begin{array}{llllll}
A_{1} & A_{22} & 0 & B_{11} & 0 & \bar{B}_{w}
\end{array}\right] \text {, } \\
& \Phi_{2}=\left[\begin{array}{lllll}
M_{2}+N_{2}-N_{2}+S_{2}-M_{2}-S_{2} & 0 & 0 & 0
\end{array}\right] \text {. }
\end{aligned}
$$

According to the condition $\sum_{2}<0$, it is proved that it is completed if it satisfies $\dot{V}_{2}(t) \leq 2 \alpha_{2} V_{2}(t)$.

Theorem 2. For a given state gain matrix $K$ and interference signal $P_{D o S}(t)$ (9), consider system (33), when the sequence satisfies $\{n T\}_{n \in N}$, and parameters $T$ and $T_{o f f}^{\min }$ are known. For known positive scalars $\alpha_{i}, \mu_{i}, h>0$ and $\gamma>0$ and the trigger parameter $0<\delta_{y}<1,0<\delta_{u}<1$, such as symmetric, positive, definite matrix, $W_{y}, W_{u}, P_{i}, Q_{i}, R_{i}$, and $Z_{i}$, and matrix $M_{i}, N_{i}, S_{i}, i \in\{1,2\}$. Both (35) and the following inequalities are satisfied:

$$
\begin{aligned}
Q_{i} & \leq \mu_{3-i} Q_{3-i}, \\
P_{1} & \leq \mu_{2} P_{2}, \\
Z_{i} & \leq \mu_{3-i} Z_{3-i}, \\
P_{2} & \leq \mu_{1} e^{2\left(\alpha_{1}+\alpha_{2}\right) h} P_{1}, \\
R_{i} & \leq \mu_{3-i} R_{3-i}, \\
0 & <\lambda=-2\left(\alpha_{1}+\alpha_{2}\right) h+2 \alpha_{1} T_{\text {off }}^{\min }-\operatorname{In}\left(\mu_{1} \mu_{2}\right) \\
& \quad-2 \alpha_{2}\left(T-T_{\text {off }}^{\min }\right) .
\end{aligned}
$$

The closed-loop system (33) under known periodic DoS interference attacks (9) is globally exponentially stable with a decay rate.

According to the judgment basis of Theorems 1 and 2, it is determined that the system is globally exponentially stable. However, there is a coupling relationship between the

corresponding matrix $\left(A_{c}, A_{c d}, K, B_{c}\right)$ and $P_{i}$ in formula (29), so the controller cannot be directly designed. Therefore, Section 5 is needed to design the controller further.

\section{Design of $H_{\infty}$ Dynamic Output Feedback Controller}

Theorem 3. For the given matrix $K$ and pulse width interference signal $P_{D o S}(t)(9)$, the sequence $\{n T\}_{n \in N}$ and $p a-$ rameters $T$ and $T_{\text {off }}^{\min }$ are known. Considering series (33), for the known positive scalar, $\alpha_{i}, h>0, \gamma>0, \gamma_{n}(n=1,2,3,4,5)$ and, double-ended elastic event trigger parameter, $0<\delta_{y}<1$ and $0<\delta_{u}<1$. If it exists in the positive definite matrix, $W_{u}, W_{y}, \widetilde{P}_{i}, \widetilde{Q}_{i}, \widetilde{R}_{i}, \widetilde{Z}_{i}, X$, and $Y$ and the matrix $\widetilde{M}_{i}, \widetilde{N}_{i}, \widetilde{S}_{i}, \quad i \in\{1,2\}, Z=\left[\begin{array}{cc}X & * \\ I & Y\end{array}\right]>0$, which is satisfied at the same time:

$$
\widetilde{\Theta}_{1}=\left[\begin{array}{cccccc}
\widetilde{\Pi}_{11}^{i} & * & * & * & * & * \\
\sqrt{h} \widetilde{N}_{i}^{T} & \widetilde{\Upsilon}_{22}^{i} & * & * & * & * \\
\sqrt{h} \widetilde{S}_{i}^{T} & 0 & \widetilde{\Upsilon}_{33}^{i} & * & * & * \\
\sqrt{h} \tilde{M}_{i}^{T} & 0 & 0 & \widetilde{\Upsilon}_{44}^{i} & * & * \\
\sqrt{h} \widetilde{R}_{i} \widetilde{A}_{i} & 0 & 0 & 0 & \widetilde{\Upsilon}_{55}^{i} & * \\
\sqrt{h} \widetilde{Z}_{i} \widetilde{A}_{i} & 0 & 0 & 0 & 0 & \tilde{\Upsilon}_{66}^{i}
\end{array}\right]<0, \quad i=1,2 .
$$

Then, the closed-loop system (33) is globally exponentially stable under the double-ended elastic event trigger 
mechanism (11) to (23). Also, the gain matrix of the output feedback controller (29) can be calculated as follows:

$$
\begin{aligned}
A_{c} & =N^{-1}\left(\Lambda_{2}-Y A_{a} X\right)(I-Y X)^{-1} N, \\
B_{c} & =N^{-1} \Lambda_{1}, \\
K & =\Lambda_{3}(I-Y X)^{-1} N, \\
A_{c d} & =N^{-1}\left(\Lambda_{4}-\Lambda_{1} C_{a} X-Y B_{a} \Lambda_{3}\right)(I-Y X)^{-1} N .
\end{aligned}
$$

Proof. The matrix $P$ is decomposed into

$$
P_{i}=\left[\begin{array}{cc}
Y & * \\
N^{T} & N^{T}\left(Y-X^{-1}\right)^{-1} N
\end{array}\right], \quad i=1,2,
$$

using Lemma 1

$$
P_{i}>0 \Leftrightarrow Z=\left[\begin{array}{cc}
X & * \\
I & Y
\end{array}\right]>0 \Leftrightarrow\left\{\begin{array}{l}
Y-X^{-1}>0, \\
X>0 .
\end{array}\right.
$$

Define the matrix:

$$
\begin{aligned}
& \Upsilon_{1}=\left[\begin{array}{cc}
X & I \\
N^{-1}(I-Y X) & 0
\end{array}\right], \\
& \Upsilon_{2}=P_{i} \Upsilon_{1}=\left[\begin{array}{cc}
I & Y \\
0 & N^{T}
\end{array}\right], \\
& \Omega=\operatorname{diag}\left\{\Upsilon_{1}, \Upsilon_{1}, \Upsilon_{1}, \Upsilon_{1}, I, I, \Upsilon_{2}, \Upsilon_{2}, \Upsilon_{2}, \Upsilon_{2}, \Upsilon_{2}\right\} .
\end{aligned}
$$

By using $\Omega$ to perform the congruent transformation of the conditions in Theorem 1, the result is obtained:

$$
\begin{aligned}
& \widetilde{\Theta}_{1}=\Omega^{T} \Theta_{1} \Omega<0, \quad i=1,2, \\
& \widetilde{\Theta}_{1}=\left[\begin{array}{cccccc}
\widetilde{\Pi}_{11}^{i} & * & * & * & * & * \\
\sqrt{h} \widetilde{N}_{i}^{T} & \widetilde{\Upsilon}_{22}^{i} & * & * & * & * \\
\sqrt{h} \widetilde{S}_{i}^{T} & 0 & \widetilde{\Upsilon}_{33}^{i} & * & * & * \\
\sqrt{h} \widetilde{M}_{i}^{T} & 0 & 0 & \widetilde{\Upsilon}_{44}^{i} & * & * \\
\sqrt{h} \widetilde{R}_{i} \widetilde{A}_{i} & 0 & 0 & 0 & \widetilde{\Upsilon}_{55}^{i} & * \\
\sqrt{h} \widetilde{Z}_{i} \widetilde{A}_{i} & 0 & 0 & 0 & 0 & \widetilde{\Upsilon}_{66}^{i}
\end{array}\right], \\
& \Theta_{1}=\left[\begin{array}{cccccc}
\Pi_{11}^{i} & * & * & * & * & * \\
\sqrt{h} N_{i}^{T} & \Upsilon_{22}^{i} & * & * & * & * \\
\sqrt{h} S_{i}^{T} & 0 & \Upsilon_{33}^{i} & * & * & * \\
\sqrt{h} M_{i}^{T} & 0 & 0 & \Upsilon_{44}^{i} & * & * \\
\sqrt{h} R_{i} \bar{A}_{i} & 0 & 0 & 0 & \Upsilon_{55}^{i} & * \\
\sqrt{h} Z_{i} \bar{A}_{i} & 0 & 0 & 0 & 0 & \Upsilon_{66}^{i}
\end{array}\right], \\
& \widetilde{\Pi}_{11}^{i}=\widetilde{\Phi}_{i 1}+\widetilde{\Phi}_{i}+\widetilde{\Phi}_{i}^{T} \text {, } \\
& \widetilde{\Upsilon}_{22}^{i}=\widetilde{\Upsilon}_{33}^{i}=-Z e^{2(-1)^{i} \alpha_{i} \lambda_{i} h} \widetilde{R}_{i} Z \text {, } \\
& \Upsilon_{44}^{i}=-Z e^{2(-1)^{i} \alpha_{i} \lambda_{i} h} \widetilde{Z}_{i} Z, \\
& \lambda_{1}=1 \text {, } \\
& \lambda_{2}=0 \text {, } \\
& \widetilde{\Phi}_{11}=\left[\begin{array}{cccccc}
\widetilde{P}_{11} & * & * & * & * & * \\
\Upsilon_{1}^{T} A_{12}^{T} P_{1} \Upsilon_{1}+\Upsilon_{1}^{T} K^{T} D_{a}^{T} C_{a 1} \Upsilon_{1} & \Upsilon_{1}^{T} H_{221} \Upsilon_{1} & * & * & * & * \\
0 & 0 & -e^{-2 \alpha_{1} h} \widetilde{Q}_{1} & * & * & * \\
B_{11}^{T} P_{1} \Upsilon_{1} & \Upsilon_{1}^{T} W_{y} C_{a} \Upsilon_{1} & 0 & \Upsilon_{1}^{T}\left(W_{y}-\delta_{y} W_{y}\right) \Upsilon_{1} & * & * \\
D_{a}^{T} C_{a 1} \Upsilon_{1}+B_{12}^{T} P_{1} \Upsilon_{1} & H_{52} \Upsilon_{1} & 0 & 0 & H_{551} & * \\
\bar{B}_{w}^{T} P_{1} \Upsilon_{1}+D_{a 1}^{T} C_{a 1} \Upsilon_{1} & D_{a 1}^{T} D_{a} K \Upsilon_{1} & 0 & 0 & D_{a 1}^{T} D_{a} D_{a 1}^{T} D_{a 1}-\gamma^{2}
\end{array}\right] \text {, }
\end{aligned}
$$




$$
\begin{aligned}
& \widetilde{P}_{11}=\Upsilon_{1}^{T}\left(P_{1} A_{1}+A_{1}^{T} P_{1}+2 \alpha_{1} P_{1}+C_{a 1}^{T} * C_{a 1}\right) \Upsilon_{1}+\widetilde{Q}_{1}, \widetilde{\Upsilon}_{55}^{i}=-\widetilde{R}_{i}, \\
& \widetilde{\Upsilon}_{66}^{i}=-\widetilde{Z}_{i}, \widetilde{Z}_{i}=\Upsilon_{2}^{T} Z_{i} \Upsilon_{2}, \widetilde{Q}_{i}=\Upsilon_{1}^{T} Q_{i} \Upsilon_{1} \text {, } \\
& \widetilde{N}_{i}=\Upsilon_{2}^{T} N_{i} \Upsilon_{2} \text {, } \\
& \widetilde{M}_{i}=\Upsilon_{2}^{T} M_{i} \Upsilon_{2} \text {, } \\
& \widetilde{S}_{i}=\Upsilon_{2}^{T} S_{i} \Upsilon_{2} \text {, } \\
& H_{551}=\delta_{u} W_{u}+W_{u}+D_{a}^{T} D_{a} \text {, } \\
& \widetilde{R}_{i}=\Upsilon_{2}^{T} R_{i} \Upsilon_{2}, \\
& H_{221}=K^{T} W_{u} K+C_{a}^{T} W_{y} C_{a}+K^{T} D_{a}^{T} D_{a} K \text {, } \\
& H_{52}=W_{u} K+D_{a}^{T} D_{a} K
\end{aligned}
$$

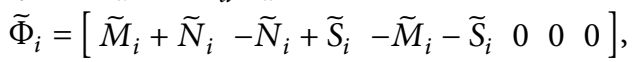

$$
\begin{aligned}
& \widetilde{A}_{1}=\left[\begin{array}{llllll}
A_{1} & A_{12} & 0 & B_{11} & B_{12} & \bar{B}_{w}
\end{array}\right] \text {, } \\
& \widetilde{\Phi}_{21}=\left[\begin{array}{cccccc}
\widetilde{P}_{22} & * & * & * & * & * \\
\Upsilon_{1}^{T} A_{22}^{T} P_{2} \Upsilon_{1} & \Upsilon_{1}^{T} H_{222} \Upsilon_{1} & * & * & * & * \\
0 & 0 & -\widetilde{Q}_{2} & * & * & * \\
B_{11}^{T} P_{2} \Upsilon_{1} & \Upsilon_{1}^{T} W_{y} C_{a} \Upsilon_{1} & 0 & \Upsilon_{1}^{T}\left(W_{y}-\delta_{y} W_{y}\right) \Upsilon_{1} & * & * \\
0 & W_{u} K \Upsilon_{1} & 0 & 0 & H_{552} & * \\
\bar{B}_{w}^{T} P_{2} \Upsilon_{1}+D_{a 1}^{T} C_{a 1} \Upsilon_{1} & 0 & 0 & 0 & 0 & D_{a 1}^{T} D_{a 1}-\gamma^{2}
\end{array}\right] \text {, } \\
& \widetilde{A}_{2}=\left[\begin{array}{llllll}
A_{1} & A_{12} & 0 & B_{11} & 0 & \bar{B}_{w}
\end{array}\right], \\
& H_{552}=-\delta_{u} W_{u}+W_{u} \text {, } \\
& H_{222}=K^{T} W_{u} K+C_{a}^{T} W_{y} C_{a} \text {, } \\
& \widetilde{P}_{22}=\Upsilon_{1}^{T}\left(P_{2} A_{1}+A_{1}^{T} P_{2}+2 \alpha_{2} P_{2}+C_{a 1}^{T} * C_{a 1}\right) \Upsilon_{1}+\widetilde{Q}_{2} .
\end{aligned}
$$

For conversions,

$$
\begin{aligned}
& \Lambda_{1}=N B_{c}, \\
& \Lambda_{2}=Y A_{a} X+N A_{c} N^{-1}(I-Y X), \\
& \Lambda_{3}=K N^{-1}(I-Y X), \\
& \Lambda_{4}=\Lambda_{1} C_{a} X+Y B_{a} \Lambda_{3}+N A_{c d} N^{-1}(I-Y X) .
\end{aligned}
$$

Applying Lemma 3 to deal with the coupling term in $\widetilde{\Upsilon}_{22}^{i}, \widetilde{\Upsilon}_{33}^{i}$, and $\widetilde{\Upsilon}_{44}^{i}$, the result is obtained:

$\operatorname{diag}\left\{\widetilde{\Upsilon}_{22}^{i}, \widetilde{\Upsilon}_{33}^{i}, \widetilde{\Upsilon}_{44}^{i}\right\}=\operatorname{diag}\left\{\gamma_{1}^{2} \widetilde{R}_{i}-2 \gamma_{1} Z, \gamma_{2}^{2} \widetilde{R}_{i}-2 \gamma_{2} Z, \gamma_{3}^{2} \widetilde{Z}_{i}-2 \gamma_{3} Z\right\}$.

The gain matrix of the controller with output feedback is

$$
\begin{aligned}
A_{c} & =N^{-1}\left(\Lambda_{2}-Y A_{a} X\right)(I-Y X)^{-1} N, \\
B_{c} & =N^{-1} \Lambda_{1}, \\
K & =\Lambda_{3}(I-Y X)^{-1} N, \\
A_{c d} & =N^{-1}\left(\Lambda_{4}-\Lambda_{1} C_{a} X-Y B_{a} \Lambda_{3}\right)(I-Y X)^{-1} N .
\end{aligned}
$$

Because equation (59) contains an unknown matrix $N$, the gain $\left(A_{c}, A_{c d}, K, B_{c}\right)$ cannot be solved directly. Therefore, through the transformation of $x_{c}(t)=N^{-1} \bar{x}_{c}(t)$, the equivalent of solution (29) is as follows:

$$
\left\{\begin{array}{l}
\dot{\bar{x}}_{c}(t)=\bar{A}_{c} \bar{x}_{c}(t)+\bar{D}_{c} \bar{x}_{c}(t-\eta(t))+\bar{B}_{c} \bar{y}_{p}(t), \\
u(t)=\bar{K} x_{c}(t), \quad t \in \psi_{k}^{m} .
\end{array}\right.
$$

The gain of the controller is expressed as follows:

$$
\begin{aligned}
\bar{A}_{c} & =\left(\Lambda_{2}-Y A_{a} X\right)(I-Y X)^{-1}, \\
\bar{B}_{c} & =\Lambda_{1}, \\
\bar{K} & =\Lambda_{3}(I-Y X)^{-1}, \\
\bar{A}_{c d} & =\left(\Lambda_{4}-\Lambda_{1} C_{a} X-Y B_{a} \Lambda_{3}\right)(I-Y X)^{-1} .
\end{aligned}
$$

\section{Example Simulation}

Considering the general satellite system model in $[26,27]$ as an example, the specific state-space model is described as (7), and the described matrix is given: 


$$
\begin{aligned}
A_{a} & =\left[\begin{array}{cccc}
0 & 0 & 1.0000 & 0 \\
0 & 0 & 0 & 1.0000 \\
-0.28 & 0.28 & -0.0043 & 0.0043 \\
0.28 & -0.28 & 0.0043 & -0.0043
\end{array}\right], \\
\Delta A_{a} & =\left[\begin{array}{cccc}
1 & 0 & 0 & 0 \\
0 & 1 & 0 & 0 \\
0 & 0 & 10 / 3 & 0 \\
0 & 0 & 0 & 10 / 3
\end{array}\right], \\
B_{a} & =\left[\begin{array}{cccc}
0 & 0 & 1 & 0.2178
\end{array}\right]^{T}, \\
B_{w} & =\left[\begin{array}{cccc}
0.1 & 0.1 & 0.1 & 0.1
\end{array}\right]^{T}, \\
C_{a} & =C_{a 1}=\left[\begin{array}{llll}
1 & 1 & 1 & 1
\end{array}\right], \\
D & =\left[\begin{array}{cccc}
0.03 & 0 & 0 & 0.01 \\
0 & 0.03 & 0 & 0.01 \\
0 & 0 & 0.03 & 0 \\
0.01 & 0.01 & 0 & 0.01
\end{array}\right], \\
E & =\left[\begin{array}{cccc}
0.01 & 0 & 0 & 0 \\
0 & 0.01 & 0 & 0 \\
0 & 0 & 0.01 & 0 \\
0 & 0 & 0 & 0.01
\end{array}\right] .
\end{aligned}
$$

Because the eigenvalue of the system matrix is $\lambda_{1}=\lambda_{2}=0, \lambda_{3}=-0.0043-0.7843 i$,

and $\lambda_{4}=-0.0043+0.7843 i$, the system is unstable without a controller.

In the following, this paper jointly designs the event trigger parameter $\left(\delta_{y}, W_{y}\right)$ and $\left(\delta_{u}, W_{u}\right)$ and the control parameter form of the event trigger state feedback, and the closed-loop system (33) is exponentially stable in periodic DoS interference attack.

Other parameter settings are as

$$
\begin{aligned}
\operatorname{miu} 1 & =\text { miu2 }=1.3, \\
\alpha_{1} & =0.2, \\
\alpha_{2} & =0.3, \\
h & =0.01, \\
\delta_{x} & =\delta_{u}=0.2, \\
T & =3, \\
T_{\text {off }}^{\min } & =2.76, \\
g g & =50, \\
\gamma_{n} & =3\left(n=1,2,3,4,5_{u}\right) .
\end{aligned}
$$

By using Matlab, the parameters of the two-terminal elastic event trigger mechanism (10), (12), (13), and (15) and the gain matrix of the controller (53) can be obtained as follows:

$$
\begin{aligned}
& \bar{K}=\left[\begin{array}{llll}
-2.3851 & 1.0982 & -5.1571 & -4.5684
\end{array}\right], \\
& W_{u}=0.5605 \text {, } \\
& W_{y}=\left[\begin{array}{cccc}
0.2083 & * & * & * \\
0.1025 & 0.0814 & * & * \\
-0.0406 & -0.0638 & 5.6805 & * \\
-0.0463 & -0.0447 & 0.1561 & 0.0239
\end{array}\right] \text {, } \\
& \bar{B}_{c}=\left[\begin{array}{c}
-0.4328 \\
0.1943 \\
1.4566 \\
-0.4108
\end{array}\right] \\
& \bar{A}_{c}=\left[\begin{array}{cccc}
0.8126 & * & * & * \\
-0.2207 & -0.1238 & * & * \\
0.3849 & -0.1134 & 3.0924 & * \\
1.4208 & 0.5436 & 1.0843 & 0.0191
\end{array}\right] \text {, } \\
& \bar{D}_{c}=\left[\begin{array}{cccc}
0.4418 & * & * & * \\
0.3056 & -1.0061 & * & * \\
-0.4158 & -0.1509 & -3.9104 & * \\
1.1845 & 0.4818 & 0.0045 & 0.0548
\end{array}\right] \text {. }
\end{aligned}
$$

The internal initial condition system is given $x_{0}=\left[\begin{array}{lll}0.1 & -0.1-0.01-0.04\end{array}\right]^{T}$, and the simulation time is assumed to be $40 \mathrm{~s}$. The state graph of DoS attack NCS based on output feedback is shown in Figure 3. Obviously, the system can have good stability through Theorem 3 .

Figures 4 and 5 show the data trigger status of the controller and sensor side under the double-ended elastic event trigger mechanism designed in this chapter. The simulation results show that 120 data on the sensor side can meet the conditions of the elastic mechanism (10) and (12), and a total of 132 sampled data on the controller side meet the conditions of the elastic trigger mechanism (13) and (15).

A total of 300 data are required to be sent to the network, and the trigger interval must be $0.1 \mathrm{~s}$. Therefore, after using the method of output feedback, the sensor has 134 data that can meet the conditions of the event mechanism.

As can be seen from Tables 1 and 2, compared with the two-terminal elastic event mechanism and periodic trigger mechanism proposed in this section, it is found that the controller and the sensor side save $55.60 \%$ and $60.00 \%$ of resources, respectively, and the transmission cycle increases by $0.1273 \mathrm{~s}$ and $0.1400 \mathrm{~s}$, respectively. Compared with the single-ended mechanism DoS attack in [28], the controller and sensor side save $4.67 \%$ and $55.60 \%$ resources, respectively, and the cycle changes are $0.0261 \mathrm{~s}$ and $0.1273 \mathrm{~s}$, respectively. Therefore, the double-ended elastic event mechanism proposed in this section meets the performance requirements of the system very well, counteracts the known periodic DoS attacks, saves limited network resources, and improves the antijamming ability of the system. 


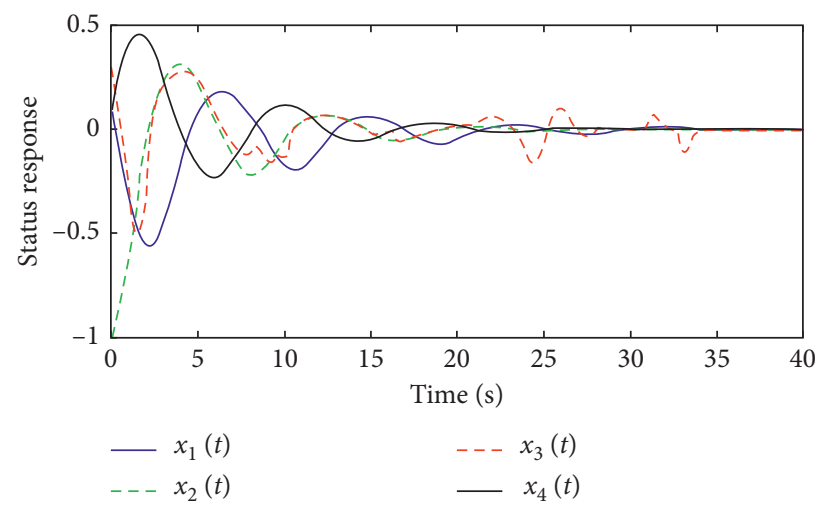

Figure 3: $T=3$ and $T_{\text {off }}^{\min }=2.76$ state response curve under output feedback.

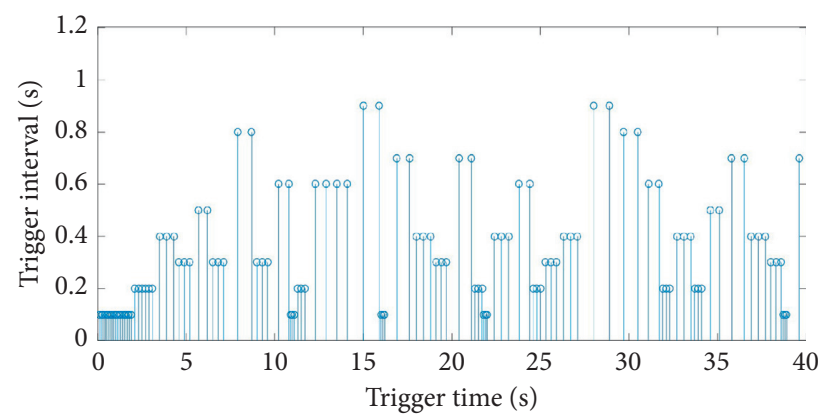

FIgURE 4: Trigger diagram of sensor side of $T=3$ and $T_{\text {off }}^{\min }=2.76$ under elastic trigger mechanism.

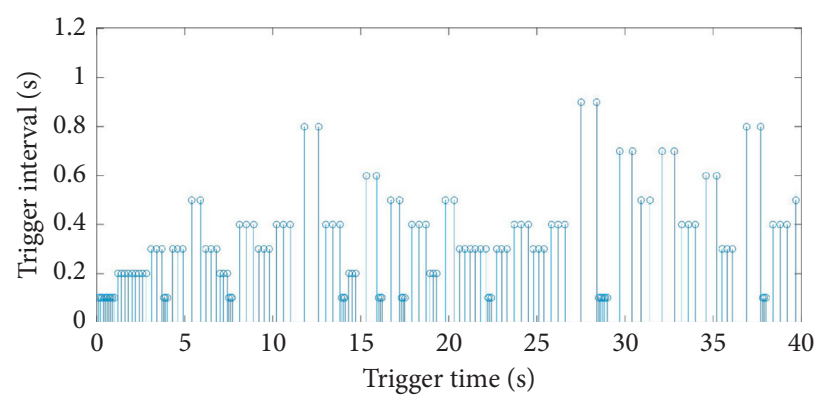

Figure 5: Controller-side trigger diagram of $T=3$ and $T_{\mathrm{off}}^{\mathrm{min}}=2.76$ under elastic trigger mechanism under output feedback.

TABle 1: Comparison diagram of output feedback data transmission rate under DoS attack based on event mechanism.

\begin{tabular}{lcc}
\hline Event trigger mechanism & Sensor side (\%) & Controller side (\%) \\
\hline Periodic trigger mechanism & 100 & 100 \\
Single-ended event mechanism DoS attack in literature [28] & 44.67 & - \\
The double-ended elastic mechanism DoS attack proposed in this chapter & 0.00 & 44.40 \\
\hline
\end{tabular}

In order to show the influence of the interference period $T$, this section also solves the following optimization problems for the time interval of different $T$ values $[0,40]$ :

$$
\Gamma_{\text {off }}^{\min }=\min \left\{T_{\text {off }}^{\min } \mid \operatorname{satisfy}(4-40)\right\} .
$$

In order to verify the influence of event trigger parameters $\delta_{x}$ and $\delta_{u}$ on system stability, Tables 3 and 4 are obtained.

To sum up, it can be concluded that (1) the system is stable; (2) the event trigger mechanism can reduce the traffic 
TABLE 2: Comparison diagram of sending cycle of output feedback data under DoS attack based on event mechanism.

\begin{tabular}{lcc}
\hline Event trigger mechanism & Sensor side (s) & Controller side (s) \\
\hline Periodic trigger mechanism & $1 / 10$ & $1 / 10$ \\
Single-ended event mechanism DoS attack in literature [28] & 0.2239 & $1 / 10$ \\
The double-ended elastic mechanism DoS attack proposed in this chapter & 0.2500 & 0.2273 \\
\hline
\end{tabular}

TABLE 3: $\lambda$ and $\omega$ come from different $T_{\text {off }}^{\min }$.

\begin{tabular}{lccccrr}
\hline$T_{\text {off }}^{\min }$ & 4.50 & 4.56 & 4.61 & 4.82 & 4.97 & 5.12 \\
\hline$\lambda$ & 0.3653 & 0.4253 & 0.4753 & 0.6853 & 0.8353 & 0.9853 \\
$\oplus$ & 0.0304 & 0.0354 & 0.0396 & 0.0571 & 0.0696 & 0.0821 \\
\hline
\end{tabular}

TABLE 4: $h_{\max }$ and $T_{\text {off }}^{\min }$ come from different $\delta_{x}$ and $\delta_{u}$.

\begin{tabular}{lccccrr}
\hline$\delta_{x}=\delta_{u}$ & 0.02 & 0.04 & 0.06 & 0.08 & 0.10 & 0.12 \\
\hline$h_{\max }$ & 0.06836 & 0.06829 & 0.06825 & 0.06821 & 0.06816 & 0.06809 \\
$T_{\text {off }}^{\min }$ & 2.76877 & 2.76883 & 2.76889 & 2.76894 & 2.76900 & 2.76905 \\
\hline
\end{tabular}

in the system; (3) the event-based output feedback controller does counteract the impact of periodic interference attacks.

\section{Conclusion}

Because the information of many objects cannot be directly monitored and there are DoS attacks in the network, this section studies the NCS under the output feedback of DoS attacks triggered by double-ended elastic events. First of all, this work proposes an elastic event trigger mechanism transmission scheme that depends on the information of the object and the controller, in order to reduce the burden of computing and communication and, at the same time, counteract the DoS interference attack imposed by the power-limited pulse width modulation (PWM) jammer. Secondly, based on the elastic event trigger mechanism and the DoS attack model, the closed-loop time-delay switching model of the system is established. Then, through LMI technology and Lyapunov knowledge, the stability criterion of $H_{\infty}$ is obtained, which contains the relationship among elastic event trigger mechanism, DoS attack, stability, and delay, and the sufficient conditions of dynamic output feedback controller and elastic event mechanism are obtained. Finally, through a numerical example, it is proved that the double-ended elastic event trigger mechanism designed in this work can not only counteract the impact of DoS attack interference and save network resources but also can better ensure the performance of the system.

\section{Data Availability}

The data used to support the findings of this study are available from the corresponding author upon request.

\section{Conflicts of Interest}

The authors declare that there are no conflicts of interest regarding the publication of this paper.

\section{Acknowledgments}

This work was supported by Science and Technology Development Program of Jilin Province under Grant no. 20180201090GX.

\section{References}

[1] B. Chen, D. W. C. Ho, G. Hu, and L. Yu, "Secure fusion estimation for bandwidth constrained cyber-physical systems under replay attacks," IEEE Transactions on Cybernetics, vol. 48, no. 6, pp. 1862-1876, 2018.

[2] L. Zha, E. Tian, X. Xie, Z. Gu, and J. Cao, "Decentralized event-triggered $\mathrm{H}_{\infty}$ control for neural networks subject to cyber-attacks," Information Sciences, vol. 457-458, no. 18, pp. 141-155, 2018.

[3] J. Liu, E. Tian, X.-P. Xie, and H. Lin, "Distributed eventtriggered control for networked control systems with stochastic cyber-attacks," Journal of the Franklin Institute, vol. 1, no. 5, pp. 48-56, 2018.

[4] J. Liu, L. Wei, X.-P. Xie, and D. Yue, "Distributed eventtriggered state estimators design for networked sensor systems with deception attacks," IET Control Theory and Applications, vol. 13, no. 9, 2014.

[5] D. Ding, Z. Wang, Q.-L. Han, and G. Wei, "Security control for discrete-time stochastic nonlinear systems subject to deception attacks," IEEE Transactions on Systems, Man, and Cybernetics: Systems, vol. 48, no. 5, pp. 779-789, 2018.

[6] H. S. Foroush and S. Martinez, "On triggering control of single-input linear systems under pulse-width modulated dos signals," SIAMJ Control Optimistic, vol. 54, no. 3, pp. 30843105, 2016.

[7] A. Y. Lu and G. H. Yang, "Input-to-state stabilizing control for cyber-physical systems with multiple transmission channels under denial-of-service," IEEE Transactions on Automatic Control, vol. 63, no. 8, pp. 1813-1820, 2018.

[8] H. Sun, C. Peng, T. Yang, H. Zhang, and W. He, "Resilient control of networked control systems with stochastic denial of service attacks," Neurocomputing, vol. 270, no. 4, pp. 170-177, 2017.

[9] L. Zha, J.-A. Fang, X. Li, and J. Liu, "Event-triggered output feedback $\mathrm{H}_{\infty}$ control for networked Markovian jump systems 
with quantizations," Nonlinear Analysis: Hybrid Systems, vol. 24, no. 6, pp. 146-158, 2017.

[10] C. D. Persis and P. Tesi, "Input-to-state stabilizing control under denial-of-service," IEEE Transactions on Automatic Control, vol. 60, no. 34, pp. 2930-2944, 2015.

[11] V. S. Dolk, P. Tesi, C. De Persis, and W. P. M. H. Heemels, "Event-triggered control systems under denial-of-service attacks," IEEE Transactions on Control of Network Systems, vol. 4, no. 1, pp. 93-105, 2017.

[12] S. Feng, P. Tesi, and C. D. Persis, "Towards stabilization of distributed systems under denial-of-service," in Proceedings of the 56th Annual Conference on Decision and Control, pp. 5360-5365, Las Vegas, NV, USA, December 2016.

[13] X. Wang and M. Lemmon, "Event design in event-triggered feedback control systems," in Proceedings of the 47th IEEE Conference on Decision and Control, pp. 2105-2110, Cancun, Mexico, December 2008.

[14] A. Eqtami, D. Dimarogonas, and K. Kyriakopoulos, "Eventtriggered control for discrete-time systems," in Proceedings of the 2010 American Control Conference, pp. 4719-4724, Baltimore, MA, USA, July 2010.

[15] R. Postoyan, A. Anta, D. Nesic, and P. Tabuada, "A unifying lyapunov-based framework for the event-triggered control of nonlinear systems," 2011, http://arxiv.org/abs/1108.5504.

[16] C. De Persis, R. Sailer, and F. Wirth, "On a small-gain approach to distributed event-triggered control," 2010, http:// arxiv.org/abs/1010.6148.

[17] C. De Persis and P. Tesi, "Networked control of nonlinear systems under Denial-of-Service," Systems \& Control Letters, vol. 96, no. 7, pp. 124-131, 2016.

[18] J. Su, R. Xu, S. Yu et al., "Redundant rule detection for software-defined networking," KSII Transactions on Internet and Information Systems, vol. 14, no. 6, pp. 2735-2751, 2020.

[19] W. Tian, X. Ji, W. Liu et al., "Defense strategies against network attacks in cyber-physical systems with analysis cost constraint based on honeypot game model," Computers, Materials \& Continua, vol. 60, no. 1, pp. 193-211, 2019.

[20] Y. Xu, T. Xu, and X. Xu, "Research on detection method of interest flooding attack on content centric network," Computers, Materials \& Continua, vol. 64, no. 2, pp. 1075-1089, 2020.

[21] J. Cheng, J. Li, X. Tang, V. S. Sheng, C. Zhang, and M. Li, “A novel DDOS attack detection method using optimized generalized multiple kernel learning," Computers, Materials \& Continua, vol. 62, no. 3, pp. 1423-1443, 2020.

[22] H. Chen and S. Kuo, "Active detecting DDOS attack approach based on entropy measurement for the next generation instant messaging app on smartphones," Intelligent Automation \& Soft Computing, vol. 25, no. 1, pp. 217-228, 2019.

[23] X.-M. Zhang and Q.-L. Han, "Event-based $\mathrm{H}_{\infty}$ filtering for sampled-data systems," Automatica, vol. 51, no. 26, pp. 55-69, 2015.

[24] C. Liu and F. Hao, "Dynamic output-feedback control for linear systems by using event-triggered quantisation," IET Control Theory \& Applications, vol. 9, no. 8, pp. 1254-1263, 2015.

[25] L. Yu, Robust Control-Linear Matrix Inequality, Tsinghua University Press, Beijing, China, 2002.

[26] B. L. Zhang, Q. L. Han, X. M. Zhang et al., "Sliding mode control with mixed current and delayed states for offshore steel jacket platform," IEEE Transactions on Control Systems Technology, vol. 22, no. 5, pp. 1769-1783, 2014.
[27] B.-L. Zhang, Q.-L. Han, and X.-M. Zhang, "Recent advances in vibration control of offshore platforms," Nonlinear Dynamics, vol. 89, no. 2, pp. 755-771, 2017.

[28] X. Chen, Y. Wang, and S. Hu, "Event-based robust stabilization of uncertain networked control systems under quantization and denial-of-service attacks," Information Sciences, vol. 459, no. 59, pp. 369-386, 2018. 